\title{
Long-Term, Selective Gene Expression in Developing and Adult Hippocampal Pyramidal Neurons Using Focal In Utero Electroporation
}

\author{
Ivan Navarro-Quiroga, ${ }^{\star}$ Ramesh Chittajallu, ${ }^{\star}$ Vittorio Gallo, and Tarik F. Haydar \\ Center for Neuroscience Research, Children's National Medical Center, Washington, DC 20010
}

An important step toward understanding brain function is the development of robust methods to molecularly perturb neurons in the developing and adult CNS. A technique of general use needs to allow targeting of specific tissues or cell types and be reasonably cost and time effective. Most important, however, is the requirement that the method itself not be deleterious to cell growth and function. To date, transgenic targeting and viral transduction techniques have been developed to elucidate the role of specific genes during development and disease. However, in the hippocampus, transgenic targeting of pyramidal neurons has been mostly hindered by the lack of specific marker genes. To date, the most widely used and successful approach for genetic manipulation of hippocampal neurons is adenoassociated viral infection, but this results in widespread gene delivery to multiple cell types (McCown et al., 1996). More recently, in utero electroporation (IUE), in which a series of voltage pulses are used to transfect neural precursor cells with plasmid DNA, has been used to elucidate the

Received Feb. 26, 2007; revised April 5, 2007; accepted April 6, 2007.

This work was supported by National Institutes of Health Grants P3OHD40677 (T.F.H. and V.G.), R01NS051852-01 (T.F.H.), R01NS045702 (V.G.), and R21NS050463 (V.G.) and by Citizens United for Research in Epilepsy (V.G.).

*I.N.-Q. and R.C. contributed equally to this work.

Correspondence should be addressed to Tarik F. Haydar at the above address. E-mail: thaydar@cnmcresearch.org.

DOI:10.1523/JNEUROSCI.0867-07.2007

Copyright $\odot$ 2007 Society for Neuroscience $\quad$ 0270-6474/07/275007-05\$15.00/0 prenatal development of striatum, cerebral cortex, and hippocampus (Miyasaka et al., 1999; Tabata and Nakajima, 2001; Nakahira and Yuasa, 2005; Gal et al., 2006). A major strength of IUE is the ability to target various brain regions by manually directing electrical pulses across specific embryonic progenitor cell populations. Two recent studies have also demonstrated that IUE can specifically label restricted cell types in the postnatal brain, including telencephalic inhibitory cells and several types of retinal neurons (Borrell et al., 2005; Matsuda and Cepko, 2007), and that this technique can be used with inducible gene expression approaches (Matsuda and Cepko, 2007).

IUE has thus emerged as a useful tool to genetically manipulate restricted cell types in vivo and may help to elucidate the molecular mechanisms underlying brain function and disease. Because the hippocampus comprises multiple cell types, each with its own particular developmental, morphological, and physiological profile, molecular access to individual cell groups in their native environment is required. Although IUE could potentially fulfill this need, no method exists for exclusive targeting of postnatal hippocampal pyramidal neurons. In addition, in order for IUE to become a viable method for postnatal studies, the functional consequences of IUE on neuronal physiology should be determined, but this has also never been done. Because electroporation requires the use of repetitive voltage pulses, it is possible that electroporation alters the developmental profile and physiological characteristics of targeted neurons. Expression of exogenous genes, for example those encoding the coral or jelly fish fluorescent proteins often used for cell labeling, may also influence the physiology of neurons in vivo. To address these possibilities, we developed a novel IUE method for selective, rapid, and efficient molecular access of differentiated hippocampal pyramidal neurons. High levels of exogenous gene transcription are maintained in this subset of neurons up to at least postnatal day 90 (P90), and protein expression was maintained at least up to P150. Combining this novel method with anatomical and electrophysiological tests on electroporated cells, here we demonstrate that focal electroporation is an efficient, specific, and innocuous method for long-term expression in hippocampal excitatory neurons.

\section{Focal in utero electroporation of} selected hippocampal subfields We targeted different pyramidal subfields of the hippocampus on embryonic day 14.5 (E14.5) by manually changing the orientation of the electrical field during electroporation with a plasmid encoding red fluorescent protein (RFP) under the control of the chicken $\beta$-actin promoter (pCAG-RFP). To efficiently transfect the hippocampal and dentate neuroepithelium, the electrodes were swept from 0 to $45^{\circ}$ during a five-pulse electroporation 
configuration using the interaural line as a reference plane of $0^{\circ}$ (Fig. $1 A$ ). Two days after electroporation, an extensive population of $\mathrm{RFP}^{+}$cells was observed in the hippocampal neuroepithelium, subventricular zone (SVZ), and developing pyramidal layers (Fig. $1 B$ ). RFP $^{+}$migrating neurons had a multipolar morphology, suggesting normal development (Nakahira and Yuasa, 2005). Analysis of brains collected at P15 confirmed that this $45^{\circ}$ electrode sweep resulted in transfection of the entire hippocampal anlage [including the dentate gyrus (DG)] (Fig. 1D). In separate experiments, electroporation using smaller sweeps ( 0 to $15^{\circ}$ and 0 to $30^{\circ}$ ) yielded preferential transfection of CA1 or CA3 subfields (Fig. 1A,E,F). A complete description of the electroporation methods is included in supplemental material (available at www.jneurosci.org).

\section{Selective transfection of pyramidal neurons}

Fifteen days after birth (i.e., $20 \mathrm{~d}$ after electroporation), brains were fixed, and a battery of interneuron markers was used to label the different populations of hippocampal interneurons. We found that no $\mathrm{RFP}^{+}$cells were colabeled with calretinin (Fig. 2A), parvalbumin (Fig. 2B), calbindin, cholecystokinin, or neuropeptide Y (data not shown). In addition, the hippocampus contralateral to the electroporation received extensive axonal projections from electroporated CA3 and CA1 pyramidal neurons, and these $\mathrm{RFP}^{+}$collateral projections were correctly arrayed in the stratum oriens and stratum radiatum (supplemental Fig. 1, available at www. jneurosci.org as supplemental material). The $\mathrm{RFP}^{+}$pyramidal cells were thus able to properly extend long-range Schaffer collaterals, an indication of appropriate differentiation and axonal outgrowth. Transfection efficiency was heterogeneous in the pyramidal cell layers and varied between 5 and $20 \%$ of the total cell nuclei within the transfected region (Figs. $1,2)(n=3)$. These cellular and anatomical results suggest that electroporated progenitors in the hippocampal neuroepithelium give rise exclusively to excitatory pyramidal neurons and that neither electroporation nor transfection alters their proper migration and morphological profile.

\section{Electrophysiological analysis of RFP $^{+}$neurons}

To corroborate the phenotype of the $\mathrm{RFP}^{+}$neurons and to more carefully examine whether either the electroporation
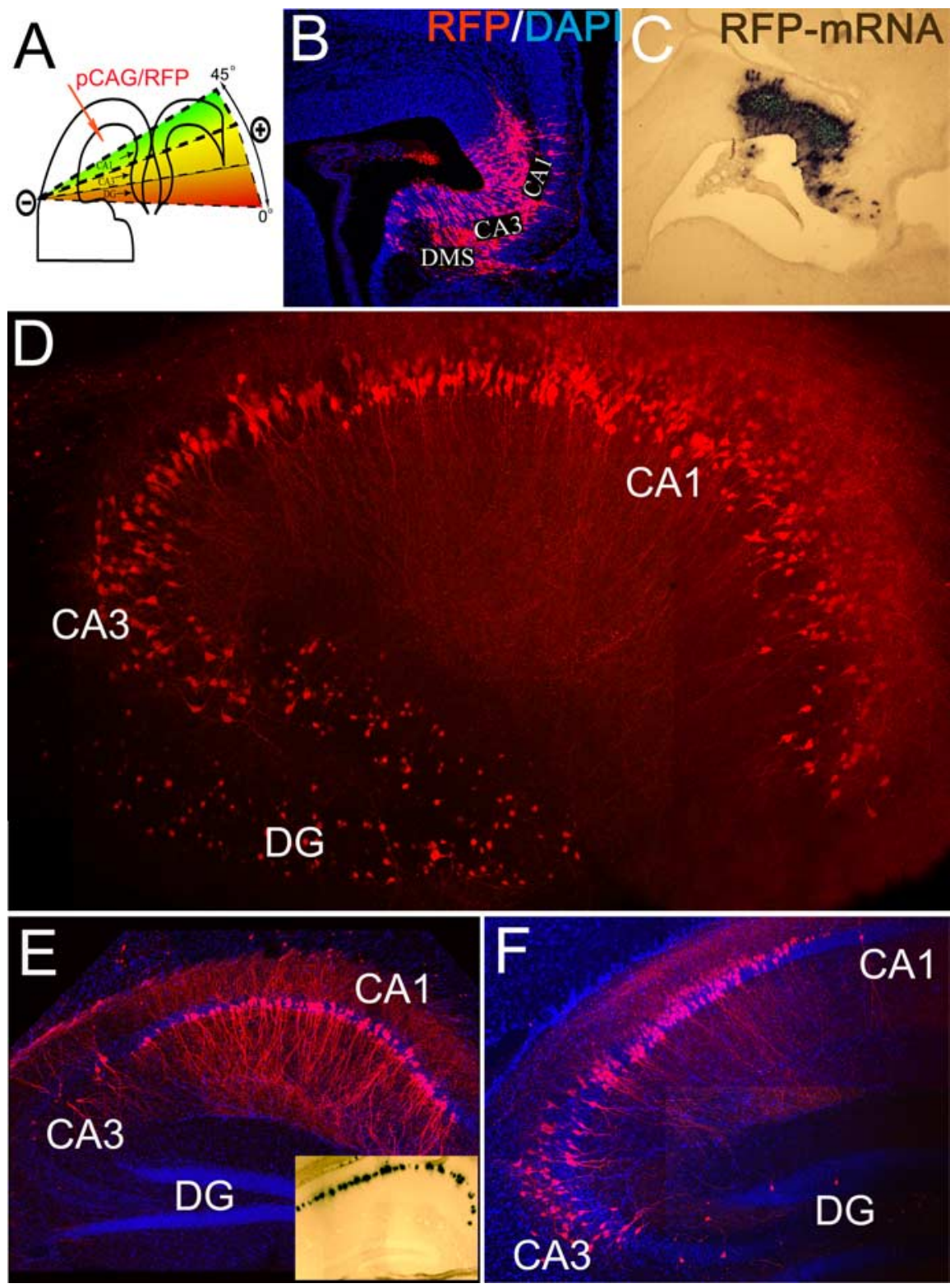

Figure 1. In utero electroporation of murine hippocampus. $A$, Diagram depicting the method for labeling specific areas of the hippocampal anlage. $\boldsymbol{B}, \boldsymbol{C}$, Two days after electroporation of CA1-CA3 and DG on E14.5, widespread RFP ( $\boldsymbol{B})$ and RFP mRNA ( $\boldsymbol{C}$ are present in the ventricular zone, SVZ, and initial differentiating fields of $C A 1-C A 3$ regions. DG precursors were also transfected, and the dentate migratory stream (DMS) was observed medially. DAPI, $4^{\prime}, 6^{\prime}$-Diamidino-2-phenylindole dihydrochloride. D, Expression of RFP in pyramidal neurons of CA1-CA3 and DG hippocampal subfields of E14.5 $\rightarrow$ P15 animals. $E, F$, Electroporation in a restricted $45^{\circ}$ plane resulted in extensive $\left(A 1\right.$ gene transfer after 1 month $(\boldsymbol{E}) . \boldsymbol{F}$, Electroporation between 15 and $45^{\circ}$ resulted in CA3 + CA1 gene transfer. E, Inset, The RFP-mRNA at P30 after electroporation on E14.5.

or the RFP expression alters functional properties of the targeted neurons, we used the whole-cell current-clamp technique to record from acutely isolated hippocampal slices from P14-P21. All RFP ${ }^{+}$ neurons ( $n=14$ cells) displayed a low frequency of action potential discharge (range, 0.7-3.2 Hz; mean \pm SEM, $2.0 \pm$ $0.24)$ during prolonged depolarizing current injections ( $+600 \mathrm{pA}$ for $60 \mathrm{~s}$ ) (Fig. $2 C)$. Using shorter subthreshold depolarizing current injections ( $+300 \mathrm{pA}$ for $1 \mathrm{~s})$, all analyzed $\mathrm{RFP}^{+}$neurons exhibited con- siderable spike frequency accommodation as highlighted by low accommodation ratio values (range, 0.08-0.37; mean \pm SEM, $0.18 \pm 0.3 ; n=14$ ) (Fig. 3B1,B3, supplemental Table 1, available at www.jneurosci.org as supplemental material). Of the $\mathrm{RFP}^{-}$cells found interspersed within the electroporated hippocampi, we clearly noted two subtypes of neurons that displayed markedly differing spike discharge profiles. Thirteen of 15 nontransfected neurons possessed spike frequency output (range, 0.8-3.0 Hz; 

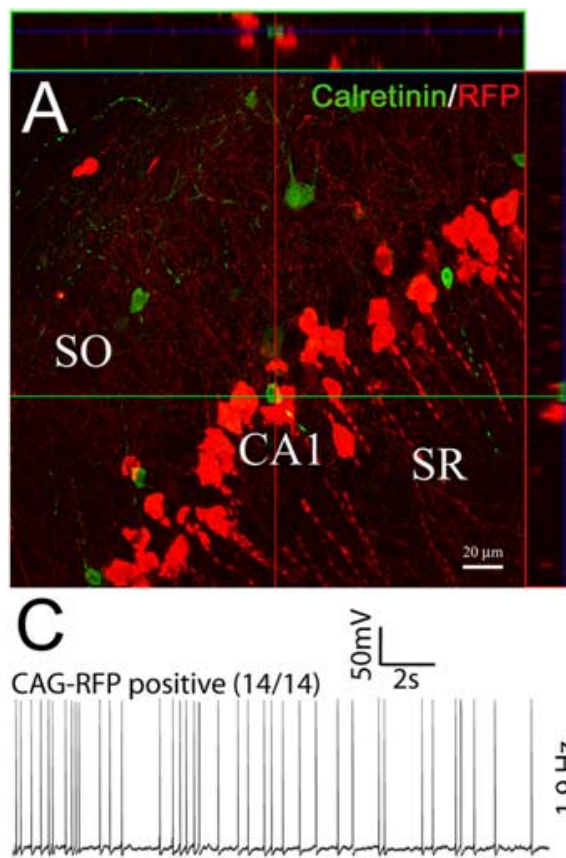

CAG-RFP negative (13/15)

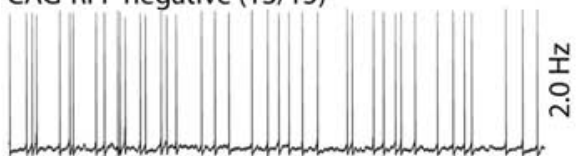

CAG-RFP negative (2/15)

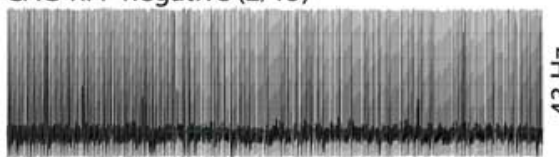

$\stackrel{N}{\mathfrak{T}}$

$\mathrm{D}$
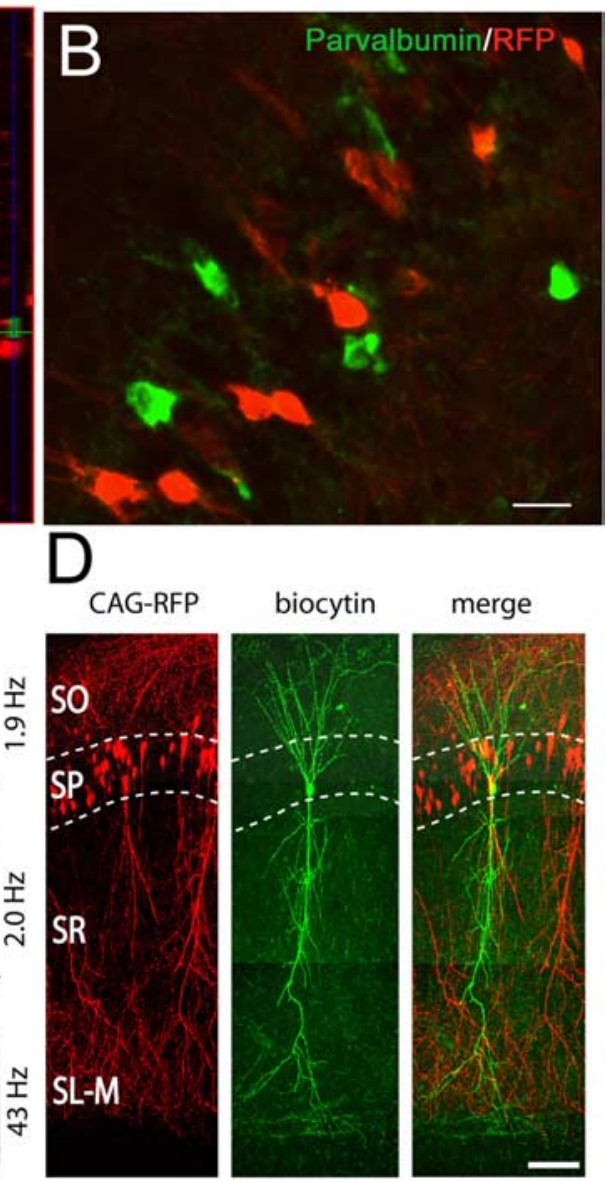
CAG-RFP
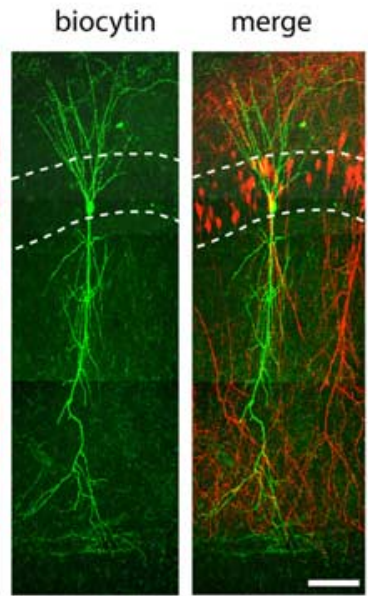

Figure 2. Electroporation on E14.5 resulted in selective transfection of pyramidal neurons. Different interneuronal markers were used to characterize RFP ${ }^{+}$cells in the pyramidal layers. $A, B, \operatorname{RFP}^{+}$neurons did not colocalize with neither calretinin $(\boldsymbol{A})$ or parvalbumin (B). C, Single examples of action potential discharge of $\mathrm{RFP}^{+}$(top) and RFP ${ }^{-}$(middle and bottom) hippocampal CA1 neurons in electroporated brain. The traces illustrate the last 20 s epoch of a 60 s suprathreshold depolarizing current injection $(+600 \mathrm{pA})$ from which the firing frequency was calculated (see values adjacent to traces). All RFP ${ }^{+}$neurons (14 of 14 cells tested) displayed low-frequency discharge, whereas RFP ${ }^{-}$neurons displayed either low ( $<4 \mathrm{~Hz} ; 13$ of 15 cells tested) or high-frequency discharge ( $>40 \mathrm{~Hz} ; 2$ of 15 cells tested). D, Morphology of an RFP-positive neuron located in the hippocampal CA1 region from which whole-cell current-clamp recording was performed. Scale bar, $100 \mu \mathrm{m}$. Note that because of dialysis and exchange of cell cytoplasm with the pipette solution during recording, varying amounts of RFP fluorescence are lost during whole-cell recording, and in many cases, it is not detected after post hoc analysis. SO, Stratum oriens; SR, stratum radiatum; SP, stratum pyramidale; SL-M, stratum lacunosam-moleculare.

mean \pm SEM, $2.2 \pm 0.31 ; n=13$ ) (Fig. $2 C)$ and spike accommodation ratios (range, 0.06-0.34; mean \pm SEM, $0.16 \pm$ $0.4 ; n=13$; data not shown) similar to the transfected neurons. However, 2 of the 15 $\mathrm{RFP}^{-}$cells displayed much higher spike frequency discharge (43 and $53 \mathrm{~Hz}$ ) (Fig. $2 C$ ) and minimal spike frequency accommodation (accommodation values of 0.87 and 0.9). From these results, we conclude that the latter neuronal group displayed the fast-spiking output characteristics of inhibitory GABAergic basket cells that are known to reside in the pyramidal layers of the hippocampus. In contrast, all cells transfected using our procedure displayed typical morphological and output charac- teristics of excitatory glutamatergic pyramidal neurons. Therefore, our functional data confirm the immunocytochemical analyses (Fig. 2A,B) and demonstrate that our novel IUE paradigm specifically targets excitatory pyramidal cells of the hippocampus.

To ascertain whether IUE and/or RFP expression alters specific functional properties of neurons, we first measured the following basic membrane parameters: (1) input resistance, (2) membrane time constant, and (3) resting membrane potential. We compared these parameters in three groups of CA1 hippocampal pyramidal neurons: (1) $\mathrm{RFP}^{+}$and (2) $\mathrm{RFP}^{-}$ controls in electroporated hippocampi and (3) control CA1 hippocampal neurons in nonelectroporated brain. In all instances, our control cells displayed lowfrequency action potential discharge and significant accommodation of spike firing and were thus classified as excitatory pyramidal neurons. Our data clearly indicate that the input resistance, membrane time constant, and resting membrane potential do not significantly differ between the three groups tested (Fig. 3A1-A3, supplemental Table 1, available at www. jneurosci.org as supplemental material). Second, we measured neuronal output properties by analyzing varying action potential firing parameters (Fig. 3B1,B2, supplemental Table 1, available at www. jneurosci.org as supplemental material). Again, no significant differences were found between spike frequency, halfwidth, amplitude, hyperpolarization amplitude, or accommodation in the three groups of pyramidal neurons tested (Fig. 3B1-B3, supplemental Table 1, available at www.jneurosci.org as supplemental material). Finally, we assessed the extent of synaptic integration by measuring miniature AMPA receptor-mediated postsynaptic currents (mEPSCs). We saw no difference in the average amplitude, decay, or frequency between any $\mathrm{RFP}^{-}$and $\mathrm{RFP}^{+}$neurons tested (Fig. 3C, supplemental Table 1, available at www. jneurosci.org as supplemental material). Thus, we conclude that neither IUE nor long-term RFP expression alters the basic membrane properties, neuronal output, and input parameters of transfected neurons.

\section{Concluding remarks and \\ future perspectives}

Gene targeting in deep structures of the adult CNS requires either stereotactic surgery or complex transgenic approaches. However, surgical approaches can cause irreparable local damage, gliosis and fibrosis, and transgenic approaches require cell- or region-specific promoters (which do not yet exist for hippocampal pyramidal neurons) and can lead to strainspecific functional and behavioral abnormalities. Among the available methods for gene delivery to adult pyramidal neurons, focal IUE takes advantage of the plasticity of the embryonic brain. Despite the relative invasiveness of the procedure, adult animals electroporated in utero display normal behavior and do not show any evident brain malformations (data not shown). The ability of this technique to specifically target pyramidal neurons via their progenitors without the require- 


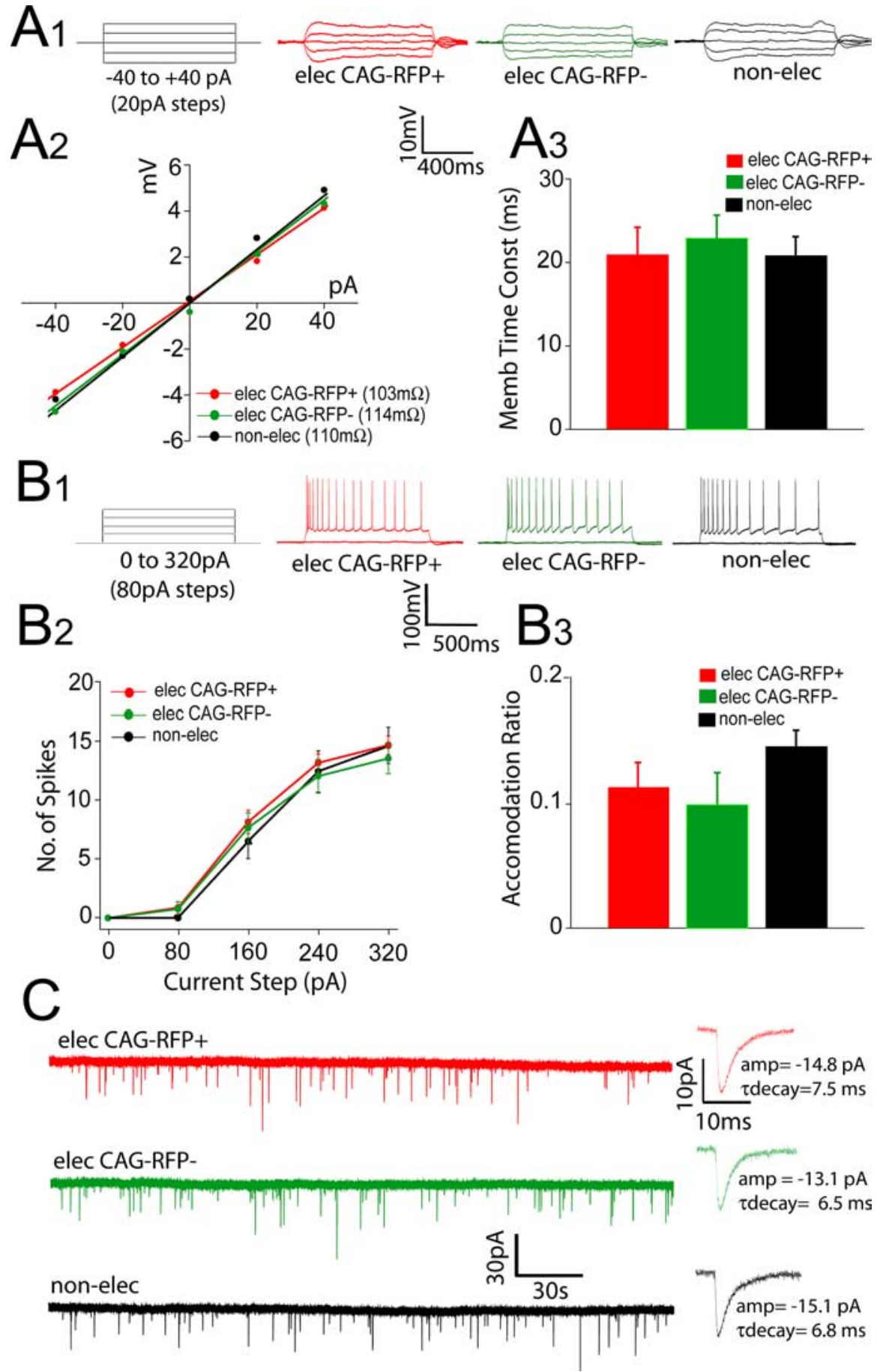

Figure 3. Comparison of functional properties between transfected and nontransfected excitatory pyramidal neurons. Throughout this figure, red and green traces depict data from RFP ${ }^{+}$and $\mathrm{RFP}^{-}$hippocampal pyramidal neurons, respectively, in mice after electroporation of pCAG-RFP as described in Materials and Methods. Black traces depict data from hippocampal pyramidal neurons from mice that had undergone the surgical procedure but without electroporation pulses. $\boldsymbol{A}$, Passive membrane responses to small subthreshold hyperpolarizing and depolarizing current injections $(\boldsymbol{A} \boldsymbol{1})$ from which the input resistances $(\boldsymbol{A} \mathbf{2})$ and membrane time constants (A3) were calculated. $\boldsymbol{B}$, Suprathreshold depolarizing current injections (B1) were used to elicit action potential discharge from which the number of spikes ( $B 2$; elicited with the range of current injections) and accommodation ratio (B3; at the $320 \mathrm{pA}$ injection) were calculated. $\boldsymbol{C}$, Sample traces and ensemble average of mEPSCs from which amplitude, frequency, and decay values were calculated. See supplemental Table 1 (available at www.jneurosci.org as supplemental material) for a summary of the comparison of all parameters measured.

ment of cell-specific promoters is a major advantage compared with transgenic targeting. From an electrophysiological standpoint, IUE is particularly advanta- geous because only a subpopulation of pyramidal neurons is transfected. Therefore, simultaneous recordings from transfected and neighboring non- transfected neurons (control) can be easily performed. It is important to note that embryonic targeting of pyramidal neuronal precursors using our technique results in persistent expression of the transgene from early developmental time points up to adulthood. Because neurotransmitters and ion channels have been shown to influence development of the embryonic brain, it will be necessary for future perturbation studies to combine our cell-type-specific labeling with an inducible system to restrict expression to the desirable time period. Future applications using electroporation of cre-inducible systems (Matsuda and Cepko, 2007) will enable temporal control of expression at different prenatal and postnatal time points.

Selective transfection of principal excitatory neurons of the hippocampal pyramidal layer is necessary for testing the role of a variety of genes under normal and pathological conditions. Synaptic plasticity in the hippocampus is a neural correlate of certain forms of learning and memory (Bliss and Collingridge, 1993; Bear and Abraham, 1996), and functional alterations in hippocampal neuronal physiology and circuit formation can result in cognitive disability. Because of the relative simplicity and the consistent high efficiency and speed of genetic manipulation, we propose that focal IUE will greatly facilitate functional studies aimed at elucidating the molecular mechanisms underlying learning and memory and human neural diseases.

\section{References}

Bear MF, Abraham WC (1996) Long-term depression in hippocampus. Annu Rev Neurosci 19:437-462.

Bliss TV, Collingridge GL (1993) A synaptic model of memory: long-term potentiation in the hippocampus. Nature 361:31-39.

Borrell V, Yoshimura Y, Callaway EM (2005) Targeted gene delivery to telencephalic inhibitory neurons by directional in utero electroporation. J Neurosci Methods 143:151-158.

Gal JS, Morozov YM, Ayoub AE, Chatterjee M, Rakic P, Haydar TF (2006) Molecular and morphological heterogeneity of neural precursors in the mouse neocortical proliferative zones. J Neurosci 26:1045-1056.

Matsuda T, Cepko CL (2007) Controlled expression of transgenes introduced by in vivo electroporation. Proc Natl Acad Sci USA 104:1027-1032.

McCown TJ, Xiao X, Li J, Breese GR, Samulski RJ (1996) Differential and persistent expression patterns of CNS gene transfer by an adenoassociated virus (AAV) vector. Brain Res 713:99-107. 
Miyasaka N, Arimatsu Y, Takiguchihayashi K (1999) Foreign gene expression in an organotypic culture of cortical anlage after in vivo electroporation. NeuroReport 10:2319-2323.

Nakahira E, Yuasa S (2005) Neuronal genera- tion, migration, and differentiation in the mouse hippocampal primoridium as revealed by enhanced green fluorescent protein gene transfer by means of in utero electroporation. J Comp Neurol 483:329-340.
Tabata H, Nakajima K (2001) Efficient in utero gene transfer system to the developing mouse brain using electroporation: visualization of neuronal migration in the developing cortex. Neuroscience 103:865-872. 